\title{
Breakage-fusion-bridge cycles and de novo telomere formation on broken chromosomes in maize callus cultures
}

\begin{tabular}{|r|l|}
\hline Journal: & Genome \\
\hline Manuscript ID & gen-2015-0211.R1 \\
\hline Manuscript Type: & Article \\
\hline Date Submitted by the Author: & $08-F e b-2016$ \\
\hline Complete List of Authors: & $\begin{array}{l}\text { Santos-Serejo, Janay; EMBRAPA Centro Nacional de Pesquisa de Mandioca } \\
\text { e Fruticultura Tropical, } \\
\text { Aguiar-Perecin, Margarida; ESALQ, University of São Paulo, Genetics }\end{array}$ \\
\hline Keyword: & $\begin{array}{l}\text { maize, heterochromatin, breakage-fusion-bridge cycle, telomere, callus } \\
\text { culture, fluorescence in situ hybridization }\end{array}$ \\
\hline
\end{tabular}




\section{Breakage-fusion-bridge cycles and de novo telomere formation on broken} chromosomes in maize callus cultures

Janay A. Santos-Serejo ${ }^{1,2}$ and Margarida L. R. Aguiar-Perecin ${ }^{1}$

${ }^{1}$ Department of Genetics, Luiz de Queiroz Agriculture College, University of São Paulo, 13418-900 Piracicaba, SP, Brazil.

${ }^{2}$ Present address: Embrapa Cassava and Fruits, Brazilian Agricultural Corporation, Cruz das Almas, BA, Brazil.

Corresponding author: Margarida L. R. Aguiar-Perecin (e-mail: mlrapere@usp.br). 


\begin{abstract}
Breakpoints involved in chromosome alterations associated with heterochromatin have been detected in maize plants regenerated from callus culture. A cytogenetic analysis of plants regenerated from a maize callus was performed aiming to analyze the stability of a chromosome 7 bearing a deficiency-duplication (Df-Dp), which was interpreted as derived from a chromatid type breakage-fusion bridge (BFB) cycle. The Df-Dp chromosome 7 was stable in mitotic and meiotic cells of the regenerated plants. Fluorescence in situ hybridization showed signals of telomeric sequences on the broken chromosome arm and provided evidence of de novo telomere formation. The stability of two types of altered chromosome 7 was investigated in C-banded metaphases from samples of the original callus that were collected during a period of 30 to 42 months after culture initiation. New alterations involving heterochromatic knobs of the chromosomes 7 and 9 were observed. The aberrant chromosomes were stable in the subcultures, thus providing evidence of broken chromosome healing. The examination of anaphases showed the presence of bridges, which was consistent with the occurrence of BFB cycles. De novo telomere formation occurred in euchromatic and heterochromatic chromosome termini. The results point to events of chromosomal evolution that might occur in plants.
\end{abstract}

Keywords: maize, heterochromatin, breakage-fusion-bridge cycle, telomere, callus culture, fluorescence in situ hybridization. 


\section{Introduction}

Changes in chromosome structure have been described in plants regenerated from tissue culture. Chromosomal breakage and its consequences (deficiencies, duplications, translocations and inversions) are events observed in callus cultures. Breakpoints involved in alterations associated with heterochromatin have been detected in maize regenerated plants (as reviewed by Peschke and Phillips 1992; Aguiar-Perecin et al. 2000). Meiotic studies of these plants have shown that most breakpoints were localized between the centromere and the heterochromatic knobs. One hypothesis that was proposed to explain the role of heterochromatin in inducing chromosome breakage was that normally late-replicating heterochromatin may replicate even later in culture, thus leading to the formation of bridges as a result of the delayed separation of chromatids at knob sites (Lee and Phillips 1987). Further studies in cells of maize embryogenic calli detected bridges resulting from delayed separation of chromatids at knob regions and typical bridges, i.e., bridges resulting from dicentric chromatids. The examination of C-banded anaphases showed that sister chromatids were held together at C-band sites (corresponding to knobs) (Fluminhan et al. 1996). Additionally, typical bridges with and without C-bands were observed. These events were interpreted as evidence of the occurrence of the chromatid type breakage-fusion-bridge (BFB) cycle initiated by chromatids that were broken during the primary event.

The first investigations on the behavior in successive nuclear divisions of a chromosome broken at meiosis showed that the chromatid type of BFB cycle initiated by broken chromosomes occurs in gametophyte mitoses and in the endosperm and that healing of broken chromosome ends occurs in the zygote (McClintock 1939, 1941). BFB cycles have been detected in other species including wheat (Triticum aestivum), in which reverse tandem duplications were observed on chromosomes (Lukaszewski 1995). The chromatid type BFB cycle initiated in meiosis continued through pollen mitoses and in early endosperm divisions but apparently did not continue in embryo mitoses. 
The healing of broken chromosomes, i.e., the addition of telomere sequences at broken ends, has been reported for diverse organisms. In plants it has been investigated by in situ hybridization using probes of telomere repetitive sequences of Arabidopsis thaliana. For example, in wheat, hybridization signals were observed at the broken arms of deleted chromosomes and at the centromeric region of telocentric chromosomes (Werner et al. 1992; Tsujimoto 1993). Studies in plants are consistent with the expression of telomerase in meristems and undifferentiated cells of in vitro cultures, but low or no detectable expression was observed in differentiated tissues (Fitzgerald et al. 1996).

In this study we carried out a cytogenetical analysis of plants regenerated from a maize callus culture, in which a deficiency-duplication (Df-Dp) on the chromosome 7 short arm was interpreted as being derived from a chromatid type BFB cycle and healing of the broken arm (Fluminhan et al. 1996). We aimed to investigate the stability of this chromosome in mitosis and meiosis of regenerated plants, including an analysis by fluorescence in situ hybridization (FISH) using probes of telomeric repeats. In addition, C-banded metaphases from samples of the original callus, collected during a period of 30 to 42 months after culture initiation, were analyzed to investigate the stability of two types of altered chromosome 7 and of other knobbed chromosomes. During this study, aberrations were detected in chromosomes 7 and 9. Mitotic instability was investigated by the examination of anaphases.

\section{Materials and methods}

\section{Plant material}

R1 and R2 generations of plants regenerated from a 12-month-old callus culture designated 3-57 were used. The plant regeneration followed the procedure described by Santos-Serejo and Aguiar-Perecin (2000). The highly embryogenic 3-57 callus was derived from the F2 hybrid $13151 \times 132131$ whose parentals were sister lines that possessed a similar knob composition: K6L2, K6L3, K7S, K7L K8L1, K8L2 and K9S (Fluminhan et al, 1996). K 
refers to knob, the number refers to the chromosome and $\mathrm{L}$ and $\mathrm{S}$ designate the long and short arms, respectively. K6L2, K6L3, K8L1 and K8L2 refer to different knob positions on chromosomes 6 and 8, according to the literature (McClintock et al., 1981). These lines were derived from the tropical flint variety Jac Duro (JD lines), and their karyotypes were characterized by Mondin et al. (2014). In this material, the chromosome 7 has a terminal knob on the short arm $(\mathrm{K} 7 \mathrm{~S})$ and an interstitial knob on the long arm $(\mathrm{K} 7 \mathrm{~L})$, but in several cells of the 3-57 culture, one altered chromosome 7 with two knobs on the short arm was observed (Fluminhan et al, 1996). According to a previous interpretation (Fluminhan et al., 1996), the two knobs on the short arm would bear a deficiency in the terminal region (Fig. S1). These knobs and the regions designated as b segment would be reverse tandem duplications (RTD) resulting from a delayed separation of sister chromatids at K7S and the formation of a bridge in anaphase, followed by chromatid breakage, a chromatid type BFB cycle and healing of the broken chromosome end.

\section{Preparation of chromosome spreads of regenerated plants}

For the somatic metaphase analysis of the regenerated plants, seeds of R1 and R2 generations were germinated at $28^{\circ} \mathrm{C}$ and excised roots were pretreated with $300 \mathrm{mg} / \mathrm{l} 8$ hydroxiquinoline for $2.5 \mathrm{~h}$, fixed in $3: 1$ ethanol:acetic acid and stored at $-20^{\circ} \mathrm{C}$. Metaphase spreads were prepared by digesting the roots in a mixture of $2 \%(\mathrm{w} / \mathrm{v})$ cellulase (final concentration 9.2 units $/ \mathrm{ml}$ ) and $0.3 \%(\mathrm{w} / \mathrm{v})$ pectinase (final concentration $14.7 \mathrm{units} / \mathrm{ml}$ ) in citrate buffer ( $\mathrm{pH} 4.6$ ). They were then immersed in $45 \%$ acetic acid (for the C-banding protocol) or in $60 \%$ acetic acid (for the FISH protocol) for 5-15 $\mathrm{min}$ and squashed. The coverslips were removed in liquid nitrogen, and the slides were air-dried and stored at $-20^{\circ} \mathrm{C}$ 
The Giemsa C-banding was performed as previously described (Bertão and AguiarPerecin 2002) to reveal the knob (C-band) composition in mitotic metaphases of the regenerated plants and of the callus subcultures.

For meiotic chromosome preparations, immature tassels from regenerated (R1 generation) and control plants were fixed in $3: 1$ ethanol:acetic acid and kept at $-20^{\circ} \mathrm{C}$. For the observation of pachytene, diakinesis and anaphase I stages, the anthers were dissected in $1 \%$ propionic carmine (prepared in 1\% propionic acid) and the microsporocytes were squashed.

\section{FISH procedure}

The FISH procedure was performed as previously reported (Mondin et al. 2007) with minor modifications. Each preparation was carried out using $20 \mu \mathrm{l}$ of probe mixture containing $15 \mathrm{ng} / \mu \mathrm{l}$ of the telomeric probe (TTTAGGG) ${ }_{6}$ directly labeled with rhodamine (Life Technologies, USA). The probe was denatured by heating at $98^{\circ} \mathrm{C}$, cooled in ice and then dropped on slide preparations onto which coverslips were applied. The preparations were denatured in a thermocycler at $83^{\circ} \mathrm{C}$ for 10 min and the hybridization was performed at $37^{\circ} \mathrm{C}$ for 16-20 h. The post-hybridization steps followed the protocol described by Mondin et al. (2007). The slides were counterstained with DAPI $(1 \mu \mathrm{g} / \mathrm{l})$ and mounted using Vectashield (Vector, USA).

\section{Subcultures of the 3-57 callus culture}

The 3-57 culture was initiated from an immature embryo as described by Fluminhan et al. (1996). Briefly, the callus was induced in MS medium (inorganic components of Murashige and Skoog 1962; $99 \mathrm{ml} / 1$ inositol, $39.4 \mathrm{mg} / \mathrm{l}$ cysteine, and vitamins according to Prioli and Silva 1989) supplemented with $2 \mathrm{mg} / 1$ 2,4-dichlorophenoxy-acetic acid (2,4D), 20 
$\mathrm{g} / \mathrm{l}$ sucrose, $20 \mathrm{mg} / \mathrm{l}$ casein hydrolysate and $8.0 \mathrm{~g} / \mathrm{l}$ agar with the $\mathrm{pH}$ adjusted to 5.8. Cultures were maintained at $26^{\circ} \mathrm{C}$ under dark conditions and were subcultured every $15-20 \mathrm{~d}$.

After 18 months of culture initiation, samples of this callus were maintained in this medium (subculture MS2) and were also transferred to MS medium supplemented with $1 \mathrm{mg} / 1$ 2,4D (subculture MS1) and to N6 medium (Chu et al. 1975) supplemented with $1.5 \mathrm{mg} / 1$ 2,4D and $1.38 \mathrm{~g} / \mathrm{l}$ proline (Armstrong and Green 1985; subculture N6). The objective was to search the best medium for the callus and to check the influence of small differences in the $2,4 \mathrm{D}$ dosage in the frequency of mitotic abnormalities. Two subcultures in each medium were maintained in different Petri dishes and were then designated as cell lines 1-MS2, 2-MS2, 1MS1, 2-MS1, 1-N6 and 2-N6, respectively.

\section{Cytogenetic analysis of the cell lines}

The cytogenetic analysis of the cell lines was performed using samples of proembryoids (globular stage) collected during a cultivation period of 30 to 42 months. Cbanded metaphases and Feulgen stained anaphases were analyzed, and the previously described procedures were employed (Fluminhan et al. 1996). Approximately 5-30 prometaphases and metaphases from each subculture were observed for the detection of alterations in chromosome 7 and in the other knobbed chromosomes. For the analysis of mitotic instability, approximately 150 anaphase cells from each sample were scored. In this analysis, very early and very late anaphases were not considered. The proportion of total abnormalities was compared by their confidence intervals with $95 \%$ of confidence using the $\mathrm{R}$ package (R Core Team, 2015).

\section{Image capture, processing and analysis of altered chromosomes}


The C-banded metaphases, Feulgen stained anaphases and carmine stained meiotic cells were photographed with Technical Pan Film (Kodak) using a Zeiss photomicroscope. FISH preparations were observed under a Zeiss Axiophot 2 microscope using the appropriate filters and the images were acquired by a CCD camera and analyzed using the ISIS software (MetaSytems, Germany). All images were processed with Adobe Photoshop 6.0.

The altered chromosomes 7 and 9 that were observed in the cell lines were measured in C-banded metaphases from the subcultures and compared with the normal chromosomes of control plants. The lengths of the short and long arms were estimated, and the centromereknob (C-band) distances in the short and long arms were also estimated. The values were presented as relative length, expressed as percent of the length of chromosome 10 (as reported by Aguiar-Perecin and Vosa 1985). The data were tested through an analysis of variance and the mean values were compared by a Tukey test using the R package (R Core Team, 2015).

\section{Results}

\section{Stability and transmission of the altered chromosome 7 in regenerated plants}

As mentioned above, in a previous analysis of the 3-57 culture (Fluminhan et al. 1996), the chromosome 7 bearing a deficiency on K7S and duplications of a b segment (DfDp 7) possessed two knobs on the short arm (Fig.S1) and was observed in most of the examined cells. Thus, plants regenerated (R0) from this culture were expected to be heterozygous for this chromosome. C-banded metaphases of root tips from $24 \mathrm{R} 1$ and 52 R2 plants obtained by self-pollination were analyzed. The Df-Dp chromosome 7 was transmitted to $45.83 \%$ and $11.76 \%$ of the plants examined, respectively. Plants that were homozygous for the aberration were not detected. It was observed that $26.9 \%$ of the $\mathrm{R} 1$ seeds did not germinate. Presumably, these seeds would be homozygous for the aberration. Fig. 1A shows a metaphase of a plant homozygous for the normal chromosome 7, and Fig. 1B shows a 
metaphase of a plant heterozygous for the aberration. Note that the distal knob (K7S) is subterminal, because there is a tiny terminal euchromatic segment in the short arm.

Telomeric signals were observed at the ends of all somatic chromosomes examined in FISH preparations, including the Df-Dp chromosome 7 (Figs. 1C-1E). Thus, this result provides evidence of telomere healing at the end of the duplicated short arm. In these DAPI stained chromosomes, bands corresponding to the knobs could be clearly visualized as reported by Mondin et al. (2014). In less condensed early metaphases, the telomeric signal was clearly detected on the euchromatic end of the short arm (Figs. 1C and 1E).

Aspects of the meiotic behavior of the regenerated plants (R1 generation) that were heterozygous for the Df-Dp chromosome 7 or homozygous for the normal chromosome were compared with those of control plants. Features of the altered chromosome were examined at the pachytene, diplotene and diakinesis stages. The duplicated short arm bearing two knobs (K7S) and the euchromatic terminal segment were observed at pachytene (Figs. 2A and 2B), while in the normal chromosome of a control plant, K7S was terminal (Fig. 2C). In the diakinesis and diplotene stages, a heteromorphic pair corresponding to chromosome 7 was observed, as expected for the heterozygotes (Figs. 2D and 2E).

The frequency of univalents at diakinesis and of laggards at anaphase I was higher in the regenerated plants than in the normal plants as shown in Table 1.

\section{BFB cycles and chromosome healing in the cell lines}

The six cell lines (1-MS2, 2-MS2, 1-N6, 2-N6, 1-MS1, 2-MS1) derived from a 18month-old sample of the 3-57 callus culture were friable embryogenic type II calli, i.e., calli with well formed embryos supported by suspensor-like structures on callus surface as previously reported for the 13233/1 callus by Fluminhan and Aguiar-Perecin (1998). The N6 subcultures (supplemented with L-proline) were more homogeneous and highly embryogenic. This medium was also used by Armstrong and Green (1985). 
The investigation of mitotic instability via analysis of Feulgen stained anaphases showed abnormalities similar to those previously described (Fluminhan et al. 1996), shown in Fig.3: a) bridges resulting from delayed separation of chromatids held together at their ends (Fig. 3A); b) broken bridges (Fig. 3B); c) typical bridges (Figs. 3C and 3D); and d) fragments (not shown). Table 2 shows the frequency of bridges and other abnormalities (broken bridges and fragments). In all cell lines, a tendency towards a decreasing frequency of abnormalities with time was observed.

Differences in the frequency of total abnormalities between cell lines cultivated in the same or in different culture media were not observed. The comparison of their confidence intervals showed overlap between cell lines, except for the subcultures 1-N6 (42 months), 1MS1 (42 months) and 2-MS1 (36 months), which presented low frequency of abnormalities and did not display overlap with some cell lines. The subculture 1-N6 (36 months) presented a higher confidence interval, probably due to the low size of the sample.

In the original 18-month-old callus culture 3-57, two types of altered chromosome 7 (Fig.4) were detected in C-banded metaphases. One of the chromosomes possessed two knobs on the short arm (K7S) as described above. The other type of aberrant chromosome 7 possessed K7S on an interstitial position of a duplicated short arm, and was previously interpreted (Fluminhan et al., 1996) as being derived from the same type of BFB cycle shown in Fig S1. In the investigated cell lines, the following types of chromosome 7 were observed (Fig. 5A): 7A, normal type; 7B, with a duplicated short arm and a subterminal K7S; 7C, with two knobs on the short arm and a terminal euchromatic segment; 7D, similar to $7 \mathrm{C}$ but without the terminal euchromatic segment; 7E, similar to 7D with a smaller terminal K7S; and 7F, with a larger short arm, a very large interstitial K7S and without the knob (K7L) on the large arm. Cells that were homozygous for the aberrant chromosomes were not observed. The 7A, 7B and 7C chromosome types were present in cells from the 18-month-old original callus culture (Fig.4). The 7C chromosome was similar to those observed in the regenerated plants 
studied here (Figs. 1B and 5E). Figs 5B and 5C illustrate the 7D and the 7B types, respectively, and the 7E type can be seen in Fig. 5 D. The $7 \mathrm{~F}$ type can be seen in the pedigree of the 1-MS1 cell line (42-month-old subculture; Fig.6). In addition, the following types of chromosome 9 were detected (Fig. 5A): 9A, normal, with a very large terminal K9S; 9B, with a smaller K9S; 9C, with a smaller subterminal K9S; 9D, without the knob; 9E, minichromosome interpreted as derived from chromosome 9, as discussed below. Fig. 5B illustrates the 9A, 9C and 9E types and the 9B type can be seen in Figs 5D and 5E. The 9D type, without knob, can be seen in Fig.6, in the 31-month-old subculture of the 1-MS1 cell line.

The cell line pedigree analysis revealed karyotype diversity among the cell lines, but homogeneity within some of them was observed in samples harvested at different age transfers (Fig.4). The number of metaphases and prometaphases observed was variable (1 to 27) depending on the mitotic index of the samples analyzed (Table S1). It is interesting to note that during this analysis, gross aberrations were not detected in the chromosomes 6 and 8 which possessed knobs, but smaller than those found on chromosomes 7 and 9.

The morphometric analysis of the different types of chromosomes 7 and 9 (Table 3) provided valuable information for the characterization of the aberrations and for the interpretation of the mechanisms of the origin of the chromosomal aberrations, as discussed below. Approximately 5-10 chromosomes of each type were evaluated. The comparison between the long arm length of the different types of chromosome 7 and the $7 \mathrm{~N}-\mathrm{C}$ (from control plants) showed that no alteration occurred, except in the 7F type, where a deficiency including the $\mathrm{K} 7 \mathrm{~L}$ was found. The short arm lengths of 7N-C and 7A types were similar, and significant differences were observed between the other types of chromosome 7. The centromere-knob (C-band) distance on the long arm $(\mathrm{CKD} / \mathrm{L})$ was similar in all types of chromosome 7. As for the distance between the centromere to the proximal knob on the short arm $(\mathrm{CKD} / \mathrm{S} 1)$, it was similar in all of the altered chromosomes compared with the 
centromere-knob distance in the normal chromosomes (7N-C and 7A). No significant differences were found between the distance of the centromere to the terminal knob $(\mathrm{CKD} / \mathrm{S}$ II) in chromosomes 7C and 7D.

The different types of chromosome 9 had a similar long arm length, except for a significant difference between 9C and 9D. Significant differences were found between the short arm lengths of the altered chromosomes (9B, 9C and 9D), and no differences were found between the centromere-knob distance in all types of chromosomes.

\section{Discussion}

Transmission and stability of the altered chromosome 7 in regenerated plants and healing of the broken short arm in callus culture

C-banded mitotic metaphases in R1 and R2 generations showed the presence of an altered chromosome 7 with two knobs (K7S) and a terminal euchromatic segment on the short arm, as well as a normal long arm with a knob (K7L). This altered chromosome was similar to the one previously observed in the 3-57 culture (Fluminhan et al., 1996)

At the pachytene stage in heterozygous R1 plants, it was also evident that the Df-Dp chromosome 7 possessed two knobs (K7S) and a terminal euchromatic segment. At diakinesis, $2.84 \%$ of the PMCs contained univalents from the heteromorphic pair corresponding to chromosome 7 . This observation and the presence of laggards at anaphase I (7.92\%) could not explain the low transmission of the altered chromosome to the R1 and R2 plants, due to their low frequency. The genotype ratios observed were not the expected according to Mendelian segregation, probably due to inviability of pollen grains or of homozygous seeds, that resulted from the presence of tandem reverse duplications on the short arm of chromosome 7. Low transmission of RTD chromosomes was also observed in wheat among progenies obtained after self-pollination of heterozygotes; this event would 
reflect reduced competitiveness of the RTDs relative to normal chromosomes on the male side (Lukaszewski, 1995).

On the other hand, the structure of the aberrant chromosome 7 was stable and similar to that observed in the original callus culture. The FISH procedure showed telomere signals at its termini in mitotic metaphases of the R1 plants. Thus, the results provide evidence that de novo telomere formation occurred at the broken chromosome end, presumably due to telomerase activity in the original 3-57 callus.

Studies in plants are consistent with the expression of telomerase in a subset of tissues, such as the meristematic tissue of cauliflower and undifferentiated cells from Arabidopsis, soybean and carrot suspensions cultures, but low or undetectable in differentiated tissues (Fitzgerald et al., 1996). In barley, differentiation and ageing resulted in a decrease in the number of telomeric repeats, whereas dedifferentiation in callus culture resulted in an increase in the number of telomeric repeats. Long-term callus cultures had very long telomeres and the absolute telomere lengths were genotype dependent (Kilian et al 1995). Interestingly, in tobacco, Fajkus et al. (1998) showed the occurrence of high telomerase activity in calli compared with leaves from which the cultures were derived and where the activity was scarcely observed. In wheat, it was demonstrated that dicentric chromosomes undergo BFB cycles in the first mitotic divisions of the gametophyte. Chromosome healing occurs during very early mitotic divisions in the sporophyte by de-novo addition of telomeric repeats, which is a gradual process until the full telomeric repeat length is acquired (Friebe et al., 2001).

In our study, we observed the addition of a new telomere at a euchromatic region, which was certainly non-telomeric, strongly suggesting telomerase expression in maize callus culture. Evidence of the healing of broken chromosomes was also observed at different sites of chromosomes 7 and 9 in long-term callus cultures as discussed below. 
The analysis of C-banded metaphases of the cell lines detected new alterations in the chromosomes 7 and 9 and the occurrence of delayed chromatid separation and bridges in anaphases provided evidence of new BFB cycle events and the healing of broken chromosomes in these long-term cultures.

The two original altered chromosomes 7 ( $7 \mathrm{~B}$ and $7 \mathrm{C}$ types) were maintained in the cultures, except for the 42-month-old subculture of the 2-N6 cell line, in which a chromosome 7 with a smaller distal K7S (7E type) was found. In addition, in the 1-MS1 and 2-MS1 cell lines, the altered chromosome 7 lost the terminal euchromatic segment (7D type). These data suggest that the cells bearing the original Df-Dp chromosome 7 (7B or 7C types) were highly adapted in culture and that the new types of chromosome 7 (7D and 7E types) detected were derived from the original altered chromosome 7 (7C type) through new events of delayed chromatid separation at the terminal knob and breakage at the knob. In addition, the chromosome 7F observed in the 42-month-old subculture of 1-MS1 is a new alteration of the normal homologue. This chromosome could have been originated through a delay in sister chromatid separation on K7S at anaphase. If the duplicated knob in sister chromatids did not separate and a breakage occurred at an adjacent euchromatic region, an amplified subterminal knob would appear. The absence of the K7L in the chromosome 7F could also be explained by a delay in the separation of the chromatids at the knob site and breakage eliminating the knob.

The morphometric analysis of chromosome 7 showed that the centromere-proximal knob distances $(\mathrm{CKD} / \mathrm{SI})$ were similar in the control and altered chromosomes. In addition, there was no difference in the distance of the centromere to the terminal knob $(\mathrm{CKD} / \mathrm{SII})$ between the chromosomes 7C and 7D. These observations and the differences in the arm lengths are consistent with the assumptions on the origin of the new chromosome types $(7 \mathrm{D}$, 7E ) that we made here. 
Chromosome 9 suffered alterations in most cell lines except for 1-MS2 and 1-N6 in which the normal chromosome (9A) was observed. In the 2-MS2 and 2-MS1 cell lines the K9S was not detected (9D type), and the 2-N6 cell line presented a partial deletion of this knob (9B type). A delayed separation of chromatids at the K9S in anaphase and a breakage that totally or partially eliminated the knob would explain the appearance of these deletions. The most interesting observation was made in the 1-MS1 cell line, in which a chromosome 9 displaying a subterminal small K9S (9C type), a chromosome 9 without knob (D type), and a minichromosome (9E type) could have resulted from the mechanism suggested in Fig.7. The primary event would be a delayed separation of chromatids at K9S, followed by breakage of the knob in one of the chromatids. In the next mitotic cycle the sister broken chromatids, fused at the knob site, would have undergone nondisjunction and given rise to a dicentric chromosome that would initiate a BFB cycle similar to the chromosomal type described by McClintock (1942). In the next mitosis, a double bridge would occur if the two centromeres of each chromatid passed to opposite poles at anaphase. The detection of double bridges supports this assumption. Among the possible products from the double bridge, two chromatids, one possessing a deficient knob and the other lacking a knob, would initiate a chromatid BFB cycle. The anaphase possessing the knob would give rise to a chromosome with a subterminal K9S (9C type) and to a knobless chromatid (9D type), both of which were observed in the 31-month old subculture. The anaphase without a knob would give rise to a chromatid from which the minichromosome (9E type) would be derived. The mechanism involved is unclear, but we suppose that the prolongation of the BFB cycle in successive nuclear divisions and deletions would generate the minichromosome. The $9 \mathrm{C}$ and $9 \mathrm{E}$ chromosome types were observed in several subcultures, thus providing evidence of healing of their broken ends.

The morphometric analyses of the various types of chromosome 9 showed differences in the length of the short arm of the altered chromosomes. Significant differences were not 
observed in the centromere-knob distance as expected according to the origin of aberrations hypothesized.

In the present study, only chromosomes 7 and 9 showed alterations resulting from the delayed separation of sister chromatids at knob sites that led to formation of bridges and chromosome breakage. The presence of large knobs in these chromosomes would contribute to the occurrence of this type of primary event. Rhoades and Dempsey (1973) reported the occurrence of a similar case of bridge formation to explain the elimination of chromatin from knob bearing chromosomes. This event occurred when the knob replication would be delayed or suppressed in the presence of B chromosomes at the second microspore division. The authors also mentioned that chromosomes possessing large knobs would be involved in this event more frequently. Furthermore, the investigation of chromosome changes through meiotic analysis of regenerated plants has shown that most aberrations also involved chromosome arms containing knobs (Lee and Phillips 1987; Benzion and Phillips 1988).

The frequency of anaphases with delayed separating chromatids was low, from $0.67 \%$ to $2 \%$ and the presence of bridges, broken bridges and fragments gave evidence of the occurrence of BFB cycles. The total abnormalities varied from $0.67 \%$ to $10 \%$. These results were quite similar to the ones observed in 5-month-old callus cultures derived from related lines (Fluminhan and Aguiar-Perecin, 1998). Interestingly, the frequency of abnormalities did not increase with time in culture; in fact they tended to decrease in each of the cell lines scored. Thus, in all probability, BFB cycles did not accumulate because broken chromosomes were healed.

The comparison of the confidence intervals did not show differences between total abnormalities of cell lines cultivated in different media. This means that the differences in the media or in 2,4D dosage used did not have a significant effect on the frequency of anaphase abnormalities. 
The presence of altered chromosomes 7 and 9 in the cells of the cultures analyzed showed that these cells were highly adapted in culture. This cytogenetical stability was also observed in callus cultures of Crepis capillaris which showed that an unbalanced karyotype may survive in culture (Sacristan, 1971) and that this capacity is dependent of the competitiveness of the cells. Conversely, regenerated plants from the MS1 cell line, bearing the minichromosome, were not recovered, likely due to its unbalanced karyotype.

In the present study, the data raise interesting questions for further investigations such as the mechanism underlying the delay in chromatid separation at knob sites, mentioned above, and the mechanism of de novo telomere formation in maize callus culture. The most mechanistic information on telomere formation is available through studies in Saccharomyces cerevisiae. In this species, new telomeres were added to non-telomeric broken chromosomes but a strong preference for telomerase action was observed at GT, TG, or GG nucleotides (reviewed by Ribeyre and Shore 2013). In wheat, the synthesis of telomere sequences could be initiated by 2-to-4-nucleotide target motifs in an r-DNA sequence localized in terminal position (Tsujimoto et al. 1999). In their review, Lamb et al. (2012) mentioned that DNA double strand breaks can be repaired by distinct pathways in eukaryotic cells, including homologous recombination, nonhomologous end joining and de novo formation of telomere at broken ends, and that spontaneous de novo telomere formation is a rare event. In our study, healing in the chromosomes 7 and 9 occurred possibly due to the presence of specific internal sequences to which telomerase were recruited.

In addition to showing altered chromosome stabilization in callus cultures, the results point to mechanisms of chromosomal evolution related to heterochromatin amount and involving BFB cycles that might occur in plants. Data accumulated in the literature suggest that structural chromosomal rearrangements are very often associated with heterochromatic regions composed of repetitive DNA, and frequently appear in heterochromatin-euchromatin borders (reviewed by Raskina et al, 2008). 


\section{Acknowledgements}

This work was supported by grants from the Fundação de Amparo à Pesquisa do Estado de São Paulo (FAPESP, Project 98/01170-5) and financial support to J.A.S.S., (Conselho Nacional de Desenvolvimento Científico e Tecnológico (CNPq) and FAPESP, No. 99/016404). We acknowledge Dr. A. A. F. Garcia for helping in the statistical analyses, and S. C. Menuzzo-Molina and C. Veríssimo for technical assistance.

\section{References}

Aguiar-Perecin, M.L.R., and Vosa, C.G. 1985. C-banding in maize II. Identification of somatic chromosomes. Heredity 54(1): 37-42. doi:10.1038/hyd.1985.6.

Aguiar-Perecin, M.L.R., Fluminhan, A., Santos-Serejo, J.A., Gardingo, J.R., Bertão, M.R., Decico, M.J.U., and Mondin, M. 2000. Heterochromatin of maize chromosomes: structure and genetic effects. Genet. Mol. Biol. 23(4), 1015-1019.doi: 10.1590/S141547572000000400047.

Armstrong, C.L., and Green C.E. 1985. Establishment and maintenance of friable, embryogenic maize callus and the involvement of L-proline. Planta 164(2):207-214.

Benzion, G., and Phillips, R.L. 1988. Cytogenetic stability of maize tissue cultures: a cell line pedigree analysis. Genome 30(3): 318-325. doi:10.1139/g88-056.

Bertão, M.R., and Aguiar-Perecin, M.L.R. 2002. Maize somatic chromosome preparation: pretreatments and genotypes for obtention of high index of metaphase accumulation. Caryologia 55 (2): 115-119. doi:10.1080/00087114.2002.10589266.

Chu, C.C., Wang, C.C., Sun, C.S., Hsu, C., Yin, K.C., Chu, C.Y., and Bi, F.Y. 1975. Establishment of an efficient medium for another culture of rice through comparative experiments on the nitrogen sources. Sci. Sinica 18: 659-668. 
Fajkus, J., Fulnecková, J, Hulánová, M., Berková, K., Ríha, K., and Matyásek, R. 1998. Plant cells express telomerase activity upon transfer to callus culture, without extensively changing telomere lengths. Mol. Gen. Genet. 260(5): 470-474. PMID:9894917.

Fitzgerald, M.S, McKnight T.D., and Shippen, D.E. 1996. Characterization and developmental patterns of telomerase expression in plants. Proc. Natl. Acad. Sci. U.S.A. 93(25): 14422-14427. PMID:8962067.

Fluminhan, A., and Aguiar-Perecin, M.L.R. 1998. Embryogenic response and mitotic instability in callus cultures derived from maize inbred lines differing in heterochromatic knob content of chromosomes. Ann. Bot. 82(5): 569-576. doi: 10.1006/anbo.1998.0710.

Fluminhan, A., Aguiar-Perecin, M.L.R, and Santos, J.A. 1996. Evidence for heterochromatin involvement in chromosome breakage in maize callus culture. Ann. Bot. 78(1): 73-81. doi:10.1006/anbo.1996.0098.

Friebe, B., Kynast, R.G., Zhang, P., Qi, L., Dhar, M., and Gill, B.S. 2001. Chromosome healing by addition of telomeric repeats in wheat occurs during the first mitotic divisions of the sporophyte and is a gradual process. Chrom. Res. 9(2):137-146. doi:10.1023/A:1009283003903.

Kilian, A., Stiff, C., and Kleinhofs, A. 1995. Barley telomeres shorten during differentiation but grow in callus culture. Proc. Natl. Acad. Sci. U.S.A. 92(21): 9555-9559.

Lamb, J.C., Shakirov, E.V., and Shippen, D.E. 2012. Plant telomeres. In Plant cytogenetics: genome structure and chromosome function. Edited by H. Bass and J. A. Birchler. Springer, New York. pp. 143-191. doi:10.1007/978-0-387-70869-0_7.

Lee, M., and Phillips, R.L. 1987. Genomic rearrangements in maize induced by tissue culture. Genome 29(1): 122-128. doi:10.1139/g87021.

Lukaszewski, A.J. 1995. Chromatid and chromosome type breakage-fusion-bridge cycles in wheat (Triticum aestivum L.). Genetics 140(3):1069-1085. PMID:7672578. 
McClintock, B. 1939. The behavior in successive nuclear divisions of a chromosome broken at meiosis. Proc. Natl. Acad. Sci. U.S.A. 25(8): 405-416.

McClintock, B.1941. The stability of broken ends of chromosomes in Zea mays. Genetics, 26(2): 234-282. PMID:17247004.

McClintock, B. 1942. The fusion of broken ends of chromosomes following nuclear fusion. Proc. Natl. Acad. Sci. U.S.A. 28(11): 458-463.

McClintock, B., Kato, T.A., and Blumenschein. 1981. A. Chromosome constitution of races of maize. Chapingo: Colegio de Postgraduados.

Mondin, M., Santos-Serejo, J.A., and Aguiar-Perecin, M.L.R. 2007. Chromosome characterization of Crotalaria juncea (L.) by chromosome banding and physical mapping of 18S-5.8S-26S and 5S rRNA gene sites. Genet. Mol. Biol. 30(1): 65-72. doi:10.1590/S1415-47572007000100013.

Mondin, M., Santos-Serejo, J.A., Bertão, M.R., Laborda, P., Pizzaia, D., and Aguiar-Perecin, M.L.R. 2014. Karyotype variability in tropical maize sister inbreds lines and hybrids compared with KYS standard line. Front. Plant Sci. 5: 544. doi: 10.3389/fpls.2014.00544.

Murashige, T., and Skoog, F. 1962. A revised medium for rapid growth and bio assays with tobacco tissue cultures. Physiol. Plant. 15(3): 473-497. doi:10.1111/j.13993054.1962.tb08052.x.

Peschke V.M., and Phillips, R.L. 1992. Genetic implications of somaclonal variation in plants. Adv. Genet. 30: 41-75. doi:10.1016/S0065-2660(08)60318-1.

Prioli, L.M., and Silva, W.J. 1989. Somatic embryogenesis and plant regeneration capacity in tropical maize inbreds. Braz. J. Genet. 12: 553-566.

R Core Team (2015). R: A language and environment for statistical computing. R Foundation for Statistical Computing, Vienna, Austria. URL https://www.R-project.org/. 
Raskina, O., Barber, J.C., Nevo, E., and Belyayev, A. 2008. Repetitive DNA and chromosomal rearrangements: speciation-related events in plant genomes. Cytogenet. Genome Res. 120(3-4): 351-357. doi:10.1159/000121084.

Rhoades, M.M., and Dempsey, E. 1973. Chromatin elimination induced by the B chromosome of maize: I. Mechanism of loss and the pattern of endosperm variegation. J. Hered. 64(1): 12-18.

Ribeyre, C., and Shore, D. 2013. Regulation of telomere addition at DNA double strand breaks. Chromosoma 122: 159-173. doi: 10.1007/s00412-013-0404-2.

Sacristan, M.D. 1971. Karyotipic changes in callus cultures from haploid and diploid plants of Crepis capillaris (L.) Wallr. Chromosoma 33(3): 273-283.

Santos-Serejo, J.A., and Aguiar-Perecin, M.L.R. 2000. Genótipos de milho com alta capacidade de embriogênese somática e regeneração de plantas obtidas a partir de calos. Scientia Agricola 57(4): 717-722.

Tsujimoto, H. 1993. Molecular cytological evidence for gradual telomere synthesis at broken chromosome ends in wheat. J. Plant Res. 106 (3): 239-244.

Tsujimoto, H., Usami, N., Hasegawa, K., Yamada, T., Nagaki, K., and Sasakuma, T. 1999. De novo synthesis of telomere sequences at the healed breakpoints of wheat deletion chromosomes. Mol. Gen. Genet. 262(4-5): 851-856. PMID:10628870.

Werner, J.E. Kota, R.S, Gill, B.S., and Endo T.R. 1992. Distribution of telomeric repeats and their role in the healing of broken chromosome ends in wheat. Genome 35(5): 844-848. doi:10.1139/g92-128. 


\section{Tables}

Table 1. Frequency of univalents at diakinesis and of laggards at anaphase I in R1 plants derived from the 3-57 culture and the respective control.

\begin{tabular}{lcccccc}
\hline \multirow{2}{*}{ Genotype } & \multicolumn{3}{c}{ Diakinesis } & \multicolumn{2}{c}{ Anaphase I } \\
\cline { 2 - 4 } & $\begin{array}{c}\text { Total } \\
\text { cells }\end{array}$ & \multicolumn{2}{c}{ 2 Univ.(\%) } & $\begin{array}{c}4 \\
\text { Univ.(\%) }\end{array}$ & $\begin{array}{l}\text { Total } \\
\text { cells }\end{array}$ & $\begin{array}{c}\text { Laggards } \\
(\%)\end{array}$ \\
\cline { 3 - 5 } 3-57 Dp-Df 7 & 352 & 2.84 & 7.39 & 0.57 & 164 & 7.92 \\
3-57 Normal 7 & 882 & - & 6.01 & & 318 & 5.03 \\
Control & 607 & - & 0.18 & & 368 & 1.90 \\
\hline *HP, heteromorphic pair; 3-57 Dp-Df 7: heterozygous for the aberrant chromosome 7; & 3-57 Normal 7: homozygous for the normal chromosome 7. Univ. , univalents.
\end{tabular}


Table 2. Frequency of anaphase abnormalities in subcultures of the cell lines.

\begin{tabular}{|c|c|c|c|c|c|c|c|c|}
\hline \multirow{2}{*}{$\begin{array}{l}\text { Cell } \\
\text { lines }\end{array}$} & \multirow[b]{2}{*}{ Months } & \multirow{2}{*}{$\begin{array}{l}\text { Total } \\
\text { cells }\end{array}$} & \multicolumn{6}{|c|}{ Anaphases with abnormalities (\%) } \\
\hline & & & DSC & Bridges & $\begin{array}{c}\text { Total } \\
\text { bridges }\end{array}$ & $\begin{array}{l}\text { Broken } \\
\text { bridges }\end{array}$ & Fragments & $\begin{array}{l}\text { Total } \\
\text { Abnormalities * }\end{array}$ \\
\hline \multirow[t]{3}{*}{ 1-MS2 } & 27 & 150 & 1.33 & 2.00 & 3.33 & 3.33 & 0.00 & $6.67(3.42-12.25)$ \\
\hline & 36 & 122 & 0.00 & 1.64 & 1.64 & 0.00 & 0.00 & $1.64(0.28-6.39)$ \\
\hline & 42 & 150 & 0.00 & 1.33 & 1.33 & 2.00 & 2.00 & $5.33(2.50-10.59)$ \\
\hline \multirow[t]{3}{*}{ 2-MS2 } & 27 & 150 & 0.67 & 2.00 & 2.67 & 2.67 & 0.00 & $5.33(2.50-10.59)$ \\
\hline & 36 & 150 & 0.00 & 1.33 & 1.33 & 1.33 & 0.67 & $3.33(1.23-8.01)$ \\
\hline & 42 & 150 & 0.00 & 1.33 & 1.33 & 0.00 & 0.00 & $1.33(0.23-5.22)$ \\
\hline \multirow[t]{3}{*}{$1-\mathrm{N} 6$} & 33 & 50 & 0.00 & 4.00 & 4.00 & 0.00 & 0.00 & $4.00(0.70-14.86)$ \\
\hline & 36 & 24 & 0.00 & 8.33 & 8.33 & 00.00 & 0.00 & $8.33(1.46-28.47)$ \\
\hline & 42 & 150 & 0.00 & 0.0 & 0.0 & 0.00 & 0.00 & $0.00(0.00-3.11)$ \\
\hline \multirow[t]{3}{*}{ 2-N6 } & 33 & 150 & 2.00 & 2.00 & 4.00 & 2.00 & 4.00 & $10.00(5.90-16.23)$ \\
\hline & 36 & 73 & 0.00 & 1.36 & 1.36 & 0.00 & 1.36 & $2.72(0.48-10.44)$ \\
\hline & 42 & 150 & 0.67 & 1.33 & 2.00 & 0.67 & 0.00 & $2.67(0.86-7.11)$ \\
\hline \multirow[t]{7}{*}{ 1-MS1 } & 28 & 50 & 0.00 & 2.00 & 2.00 & 2.0 & 0.00 & $4.00(0.70-14.86)$ \\
\hline & 30 & 100 & 0.00 & 1.00 & 1.00 & 0.00 & 2.00 & $3.00(0.78-9.15)$ \\
\hline & 31 & 150 & 2.00 & 1.33 & 3.33 & 3.33 & 2.00 & $8.67(4.88-14.66)$ \\
\hline & 32 & 150 & 0.00 & 2.00 & 2.00 & 3.33 & 2.67 & $8.00(4.39-13.86)$ \\
\hline & 33 & 150 & 1.33 & $3.33 \mathrm{e}$ & 4.67 & 1.33 & 2.00 & $8.00(4.39-13.86$ \\
\hline & 36 & 150 & 0.67 & 0.67 & 1.33 & 0.67 & 0.00 & $2.00(0.52-6.19)$ \\
\hline & 42 & 150 & 0.00 & 0.00 & 0.00 & 0.00 & 1.33 & $1.33(0.23-5.23)$ \\
\hline \multirow[t]{3}{*}{ 2-MS1 } & 29 & 150 & 1.33 & 2.67 & 4.00 & 1.33 & 0.67 & $6.00(2.96-11.42)$ \\
\hline & 36 & 150 & 0.67 & 0.67 & 1.34 & 0.00 & 0.00 & $1.34(2.31-5.23)$ \\
\hline & 42 & 150 & 0.00 & 0.67 & 0.67 & 0.00 & 0.00 & $0.67(0.03-4.21)$ \\
\hline
\end{tabular}

Note. DSC, delayed separating chromatids.

*Confidence intervals with $95 \%$ of confidence in brackets. 
Table 3. Relative lengths of the long (L) and short (S) arms and centromere-knob distance in the long $(\mathrm{CKD} / \mathrm{L})$ and in the short arms $(\mathrm{CKD} / \mathrm{S})$ of chromosomes 7 and 9 of the cell lines and control plants.

\begin{tabular}{|c|c|c|c|c|c|}
\hline $\begin{array}{c}\text { Chromosome } \\
\text { type }\end{array}$ & $\mathrm{L}$ & $\mathrm{S}$ & $\mathrm{CKD} / \mathrm{L}$ & $\mathrm{CKD} / \mathrm{SI} \uparrow$ & $\mathrm{CKD} / \mathrm{SII}$ \\
\hline $7 \mathrm{~N}-\mathrm{C}^{*}$ & $103.00 \mathrm{a}$ & $55.00 \mathrm{~d}$ & $55.58 \mathrm{a}$ & $38.00 \mathrm{a}$ & \\
\hline $7 \mathrm{~A}$ & $100.52 \mathrm{a}$ & $57.92 \mathrm{~d}$ & $57.48 \mathrm{a}$ & $38.08 \mathrm{a}$ & \\
\hline $7 \mathrm{~B}$ & $100.00 \mathrm{a}$ & $87.25 \mathrm{c}$ & $55.00 \mathrm{a}$ & $41.75 \mathrm{a}$ & \\
\hline $7 \mathrm{C}$ & $101.60 \mathrm{a}$ & $123.80 \mathrm{a}$ & $55.00 \mathrm{a}$ & $39.00 \mathrm{a}$ & $95.60 \mathrm{a}$ \\
\hline $7 \mathrm{D}$ & $98.16 \mathrm{a}$ & $108.20 \mathrm{~b}$ & $58.88 \mathrm{a}$ & $36.56 a$ & $88.04 \mathrm{a}$ \\
\hline $7 F$ & $62.00 \mathrm{~b}$ & $86.00 \mathrm{c}$ & - & $35.57 \mathrm{a}$ & \\
\hline $9 \mathrm{~N}-\mathrm{C}^{*}$ & $62.08 \mathrm{ab}$ & $68.42 \mathrm{a}$ & - & $39.92 \mathrm{a}$ & \\
\hline $9 \mathrm{~A}$ & $66.21 \mathrm{ab}$ & $70.68 \mathrm{a}$ & - & $40.88 \mathrm{a}$ & \\
\hline $9 B$ & $64.13 \mathrm{ab}$ & $57.73 \mathrm{c}$ & - & $40.31 \mathrm{a}$ & \\
\hline $9 \mathrm{C}$ & $67.71 \mathrm{a}$ & $65.64 a$ & - & $39.36 \mathrm{a}$ & \\
\hline $9 \mathrm{D}$ & $59.80 \mathrm{~b}$ & $45.20 \mathrm{~b}$ & - & - & \\
\hline
\end{tabular}

*7N-C and 9N-C: normal chromosomes of control plants; $\uparrow$ centromere-knob distance referring to the subterminal knob of the altered chromosome 7, terminal knob of normal 7 and terminal knob of chromosome 9 ; $\$$ centromere-knob distance referring to the terminal knob of the altered chromosome 7.

Note. Data are expressed as percent of chromosome 10. Values with the same letter are not significantly different at the level of $5 \%$ based on a Tukey test. 


\section{Figure captions}

Fig. 1. Somatic chromosomes of R1 plants. C-banded mitotic metaphases of: homozygote for normal chromosome 7 showing knobs (C-bands) on the long and short arms (A); heterozygote for the Df-Dp chromosome 7 with two knobs on the short arm (an amplification of pair 7 is shown in the square) (B); telomeric FISH signals on early and full metaphases (C, D); homologous pairs of chromosome 7 (E). Note the FISH signal on the terminus of the Df-Dp chromosome 7 short arm. Scale bar $=10 \mu \mathrm{m}$.

Fig. 2. Carmine-stained meiotic chromosomes of R1 plants heterozygous for the Df-Dp chromosome 7: pachytene of the heterozygote and respective graphic interpretation (A and B) and normal chromosome 7 of a control plant (C); diakinesis (D) and diplotene (E) chromosomes showing a heteromorphic pair (arrows). Note the presence of the euchromatic segment at the end of the short arm of the Df-Dp chromosome 7 in (A) and (B). K, knob; L, long arm; S, short arm; Cent, centromere. Scale bar $=10 \mu \mathrm{m}$.

Fig. 3. Feulgen stained anaphase cells of the cell lines with a laggard chromosome showing delayed separating chromatids (A), a broken bridge (after the primary event) (B), a typical bridge (C) and a double bridge (D). Scale bar $=10 \mu \mathrm{m}$

Fig. 4. Cell line pedigree analysis showing the types of chromosomes 7 and 9 observed in Cbanded metaphases in different subcultures of the six cell lines.

Fig. 5. Types of chromosomes 7 and 9 observed in C-banded metaphases of the cell lines (A), a metaphase cell of the 1-MS1 cell line (B) and 1-MS2 (C), and chromosomes 7 and 9 of the 2-N6 cell line (D and E). Scale bar $=10 \mu \mathrm{m}$.

Fig. 6. Types of chromosomes 7 and 9 observed in C-banded metaphases of the 1-MS1 cell line. Scale bar $=10 \mu \mathrm{m}$.

Fig. 7. Mechanism that would originate different types of chromosome 9 in the 1-MS1 cell line: normal chromosome (A); anaphase with delayed separating chromatids and breakage at K9S (B); the resulting chromatids, one normal and the other with a deficient knob (C) fused 
after replication (D); nondisjunction of sister chromatids (E) resulting in a dicentric chromosome $(\mathrm{F}, \mathrm{G})$; double bridge $(\mathrm{H})$ giving rise to a chromatid with a deficiency at K9S and another chromatid without a knob (I), which, after duplication and fusion (J), suffered breakage in the next anaphases $(\mathrm{K})$; resultant chromosomes: one with a subterminal deficient K9S, another without the knob and a minichromosome originated after further deletions whose mechanism is unclear (L). Arrows at the anaphases indicate breakpoints. 


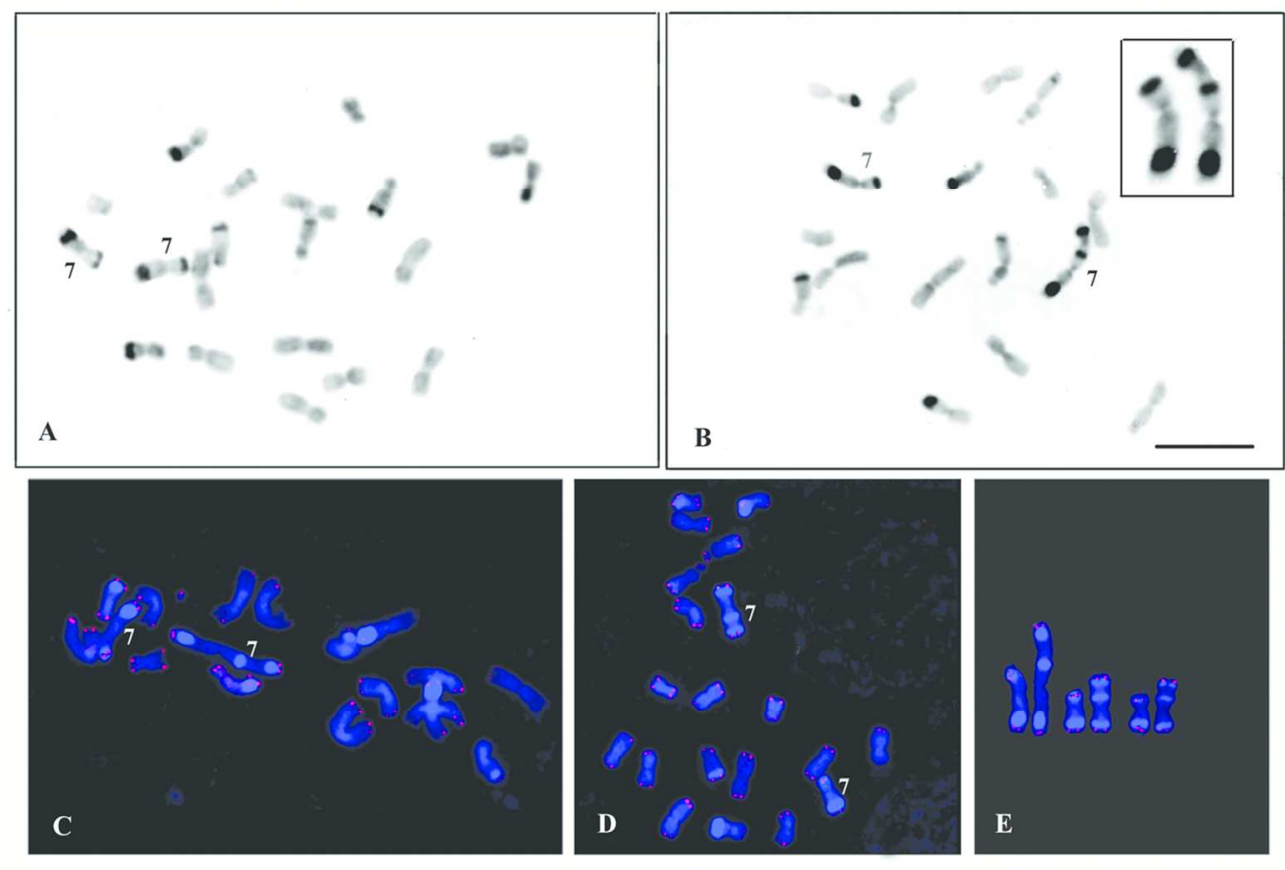

$107 \times 86 \mathrm{~mm}(300 \times 300 \mathrm{DPI})$ 

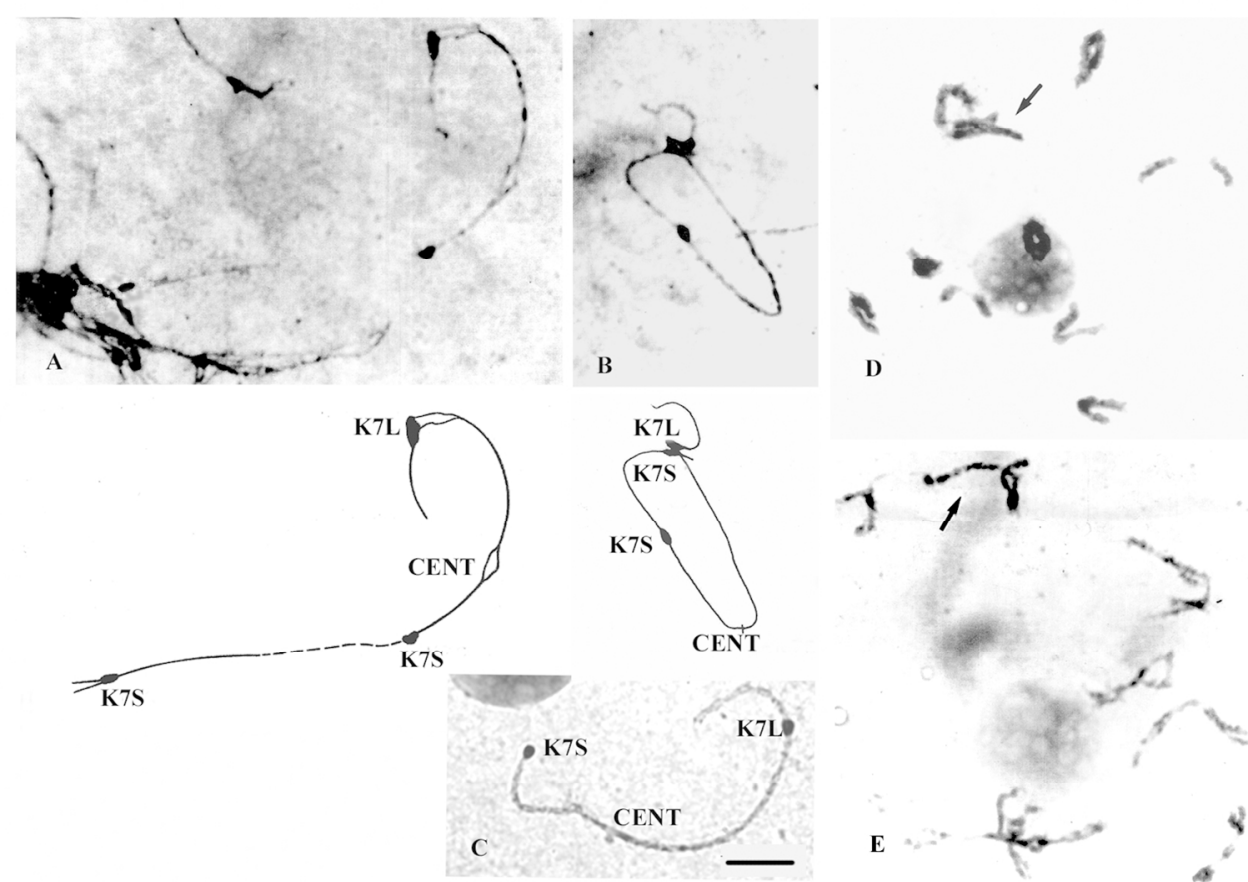

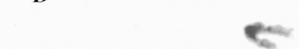

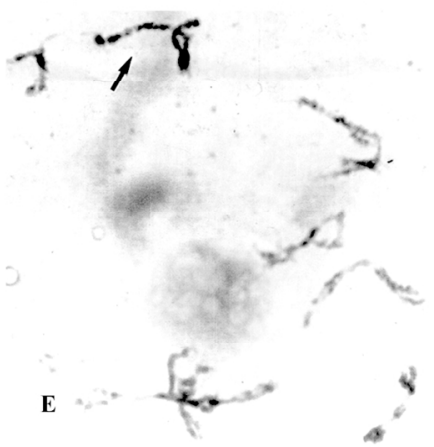

$145 \times 102 \mathrm{~mm}(300 \times 300$ DPI $)$ 

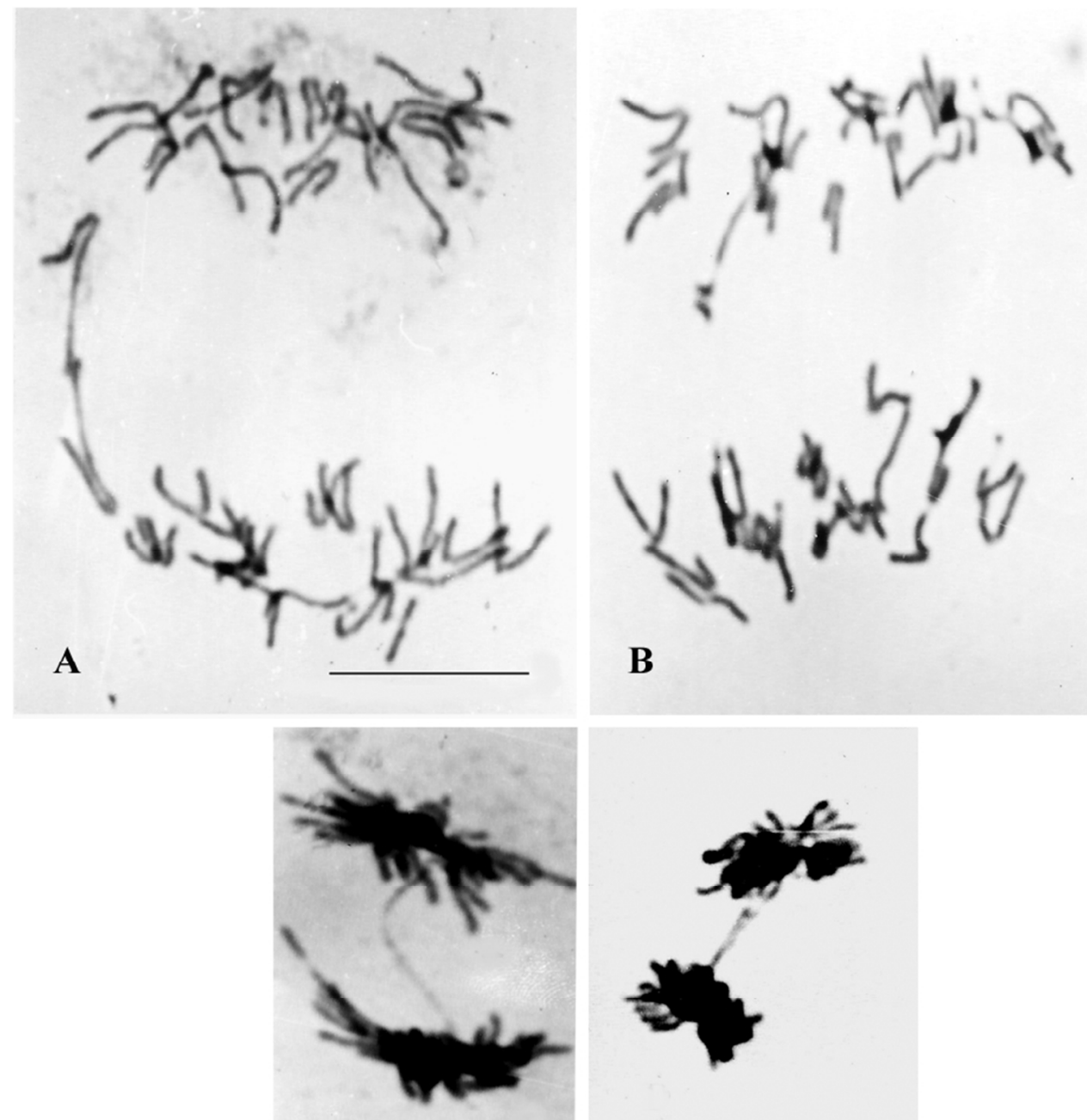

B

C

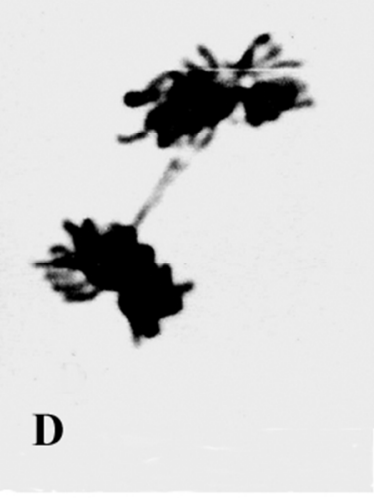

$80 \times 87 \mathrm{~mm}(300 \times 300 \mathrm{DPI})$ 


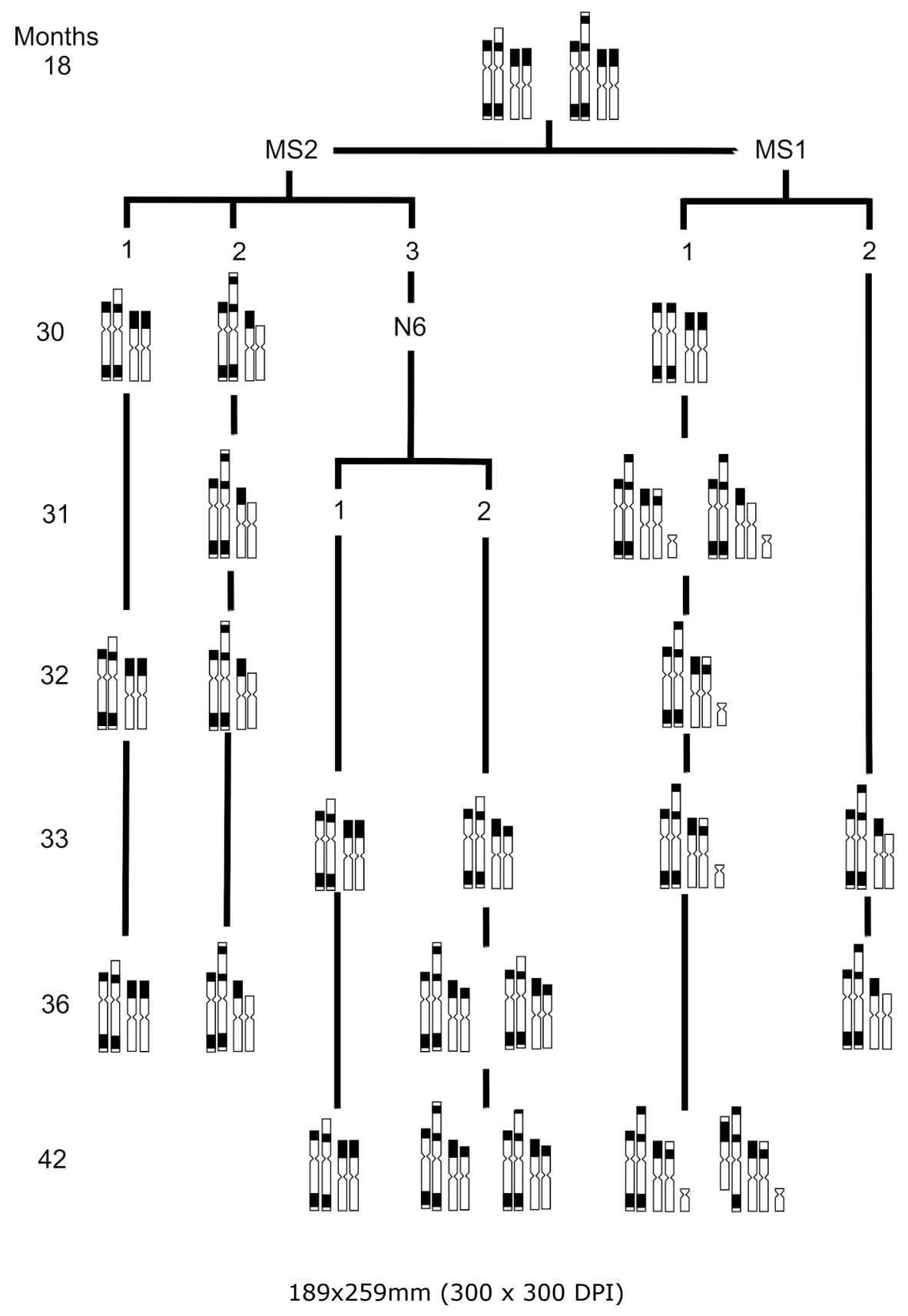




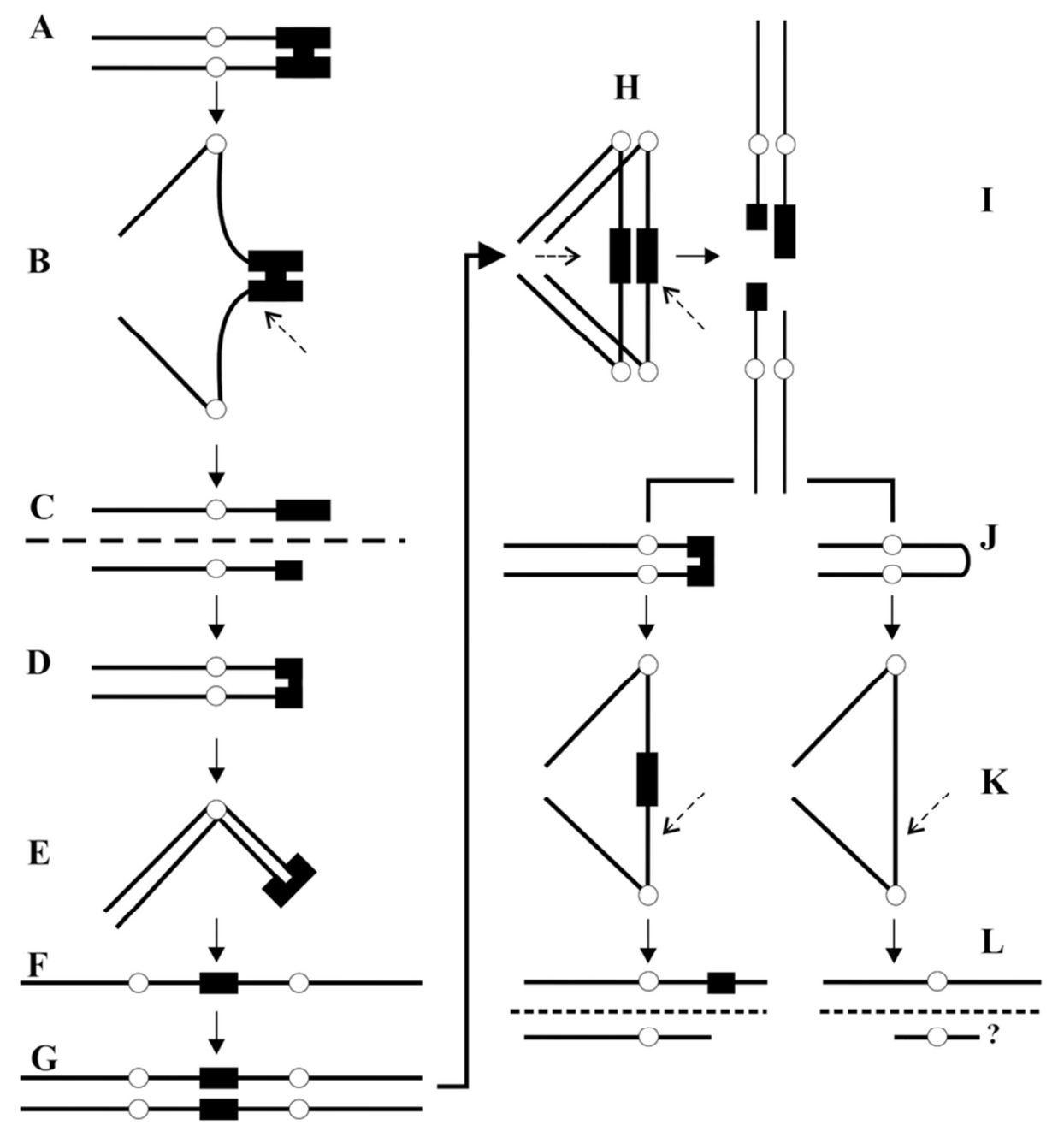

$83 \times 86 \mathrm{~mm}(300 \times 300 \mathrm{DPI})$ 
Table S1. Number of metaphases (M) and prometaphases (P) with normal and altered chromosomes 7 and 9 analyzed in the cell lines.

\begin{tabular}{|c|c|c|c|c|c|}
\hline \multirow{3}{*}{$\begin{array}{c}\text { Cell Line } \\
\text { 1-MS2 }\end{array}$} & \multirow{3}{*}{$\begin{array}{c}\begin{array}{c}\text { Age } \\
\text { (Months) }\end{array} \\
30\end{array}$} & \multicolumn{2}{|c|}{ Number } & \multirow{2}{*}{\multicolumn{2}{|c|}{$\begin{array}{c}\text { Chromosome } \\
\text { type }\end{array}$}} \\
\hline & & \multirow{2}{*}{$\begin{array}{c}\mathrm{M} \\
1\end{array}$} & \multirow[t]{2}{*}{$\mathrm{P}$} & & \\
\hline & & & & $7 \mathrm{~A} 7 \mathrm{~B}$ & 9A9A \\
\hline & 32 & 3 & 1 & 7A7B & 9A9A \\
\hline & 36 & 2 & - & 7A7B & 9A9A \\
\hline \multirow[t]{4}{*}{ 2-MS2 } & 30 & 1 & - & 7A7C & 9A9D \\
\hline & 31 & 6 & - & 7A7C & 9A9D \\
\hline & 32 & 2 & - & 7A7C & 9A9D \\
\hline & 36 & 1 & - & 7A7C & 9A9D \\
\hline \multirow[t]{2}{*}{ 1-N6 } & 33 & 7 & 3 & 7A7B & 9A9A \\
\hline & 42 & 4 & - & 7A7B & 9A9A \\
\hline \multirow[t]{5}{*}{$2-\mathrm{N} 6$} & 33 & 6 & 3 & 7A7B & 9A9B \\
\hline & 36 & 4 & 1 & 7A7B & 9A9B \\
\hline & & 1 & - & 7A7C & 9A9B \\
\hline & 42 & 12 & 9 & 7A7C & 9A9B \\
\hline & & 2 & 2 & 7A7E & $9 A 9 B$ \\
\hline \multirow[t]{7}{*}{ 1-MS1 } & 30 & 1 & - & 7A7A & 9A9A \\
\hline & 31 & 9 & 5 & 7A7D & 9А9С9E \\
\hline & & 5 & 6 & 7A7D & 9A9D9E \\
\hline & 32 & 27 & - & 7A7D & 9A9C9E \\
\hline & 33 & - & 1 & 7A7D & 9А9С9Е \\
\hline & 42 & 16 & 6 & 7A7D & 9A9C9E \\
\hline & & 16 & 2 & 7F7D & 9А9С9E \\
\hline \multirow[t]{2}{*}{ 2-MS1 } & 33 & 2 & 2 & 7A7D & 9A9D \\
\hline & 36 & 1 & - & 7A7D & 9A9D \\
\hline
\end{tabular}


https://mc06.manuscriptcentral.com/genome-pubs 


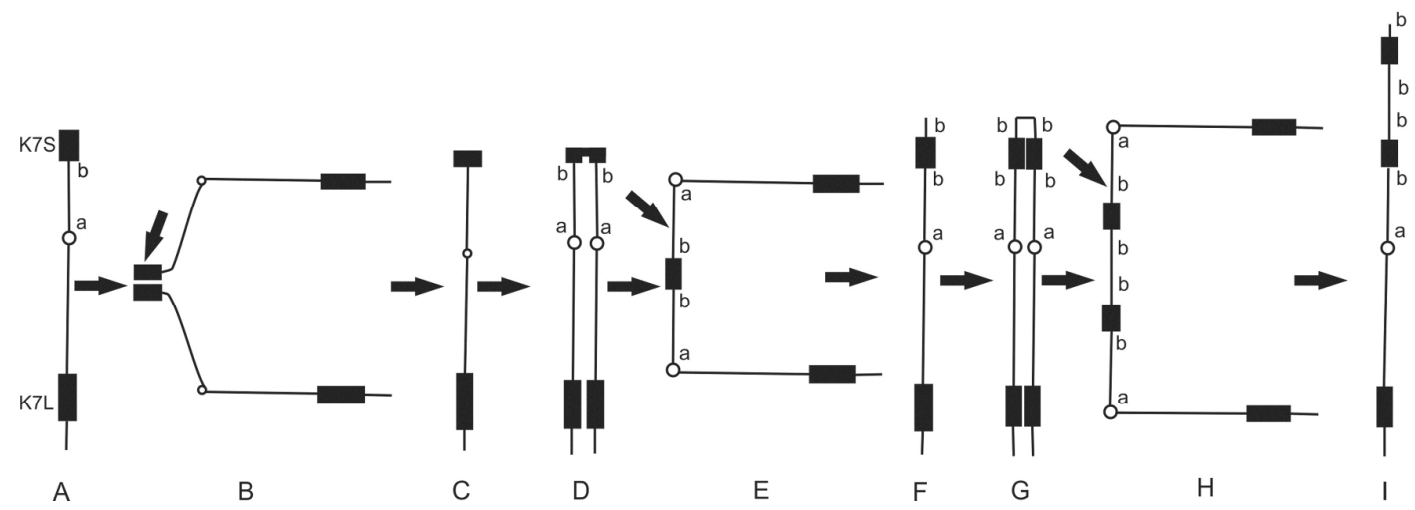

Fig. S1. BFB cycle that would give rise to a chromosome 7 bearing a deficiency and duplication (Df-Dp) showing: normal chromosome (A); anaphase with delayed separating chromatids and breakage at K7S (B); chromatid with a deficient K7S (C) fused after replication (D); breakage at anaphase (E); chromatid with duplication of the $b$ segment $(F)$ fused at broken ends after replication $(G)$; anaphase bridge and breakage $(\mathrm{H})$; and the resulting chromatid with two knobs and tandem reverse duplications (RTD) of a b segment. Arrows at the anaphases indicate breakpoints. 